\title{
Thermal study of fluid flow inside an annular pipe filled with porous media under local thermal non-equilibrium condition
}

\author{
Abdelkrim Bouaffane $^{1^{*}}$. Kamel Talbi ${ }^{1}$ \\ ${ }^{1}$ Laboratoire d'Energétique Appliquée et de Pollution, Département de Génie Mécanique, \\ Faculté des Sciences de la Technologie, Université des Frères Mentouri, \\ Constantine, Algeria \\ Phone: +213696207466 \\ *Email: a.bouafane@yahoo.fr
}

\begin{abstract}
The present work involves the thermal numerical simulation of fluid flow inside an annular pipe completely filled porous material. The mathematical model of the energy transport is based on the Local Thermal Non-Equilibrium (LTNE) between the fluid and the solid phases. The governing equations are discretized by the finite volume method. SIMPLE algorithm has been used to solve the set of algebraic discretized coupled equations. This work is divided in two parts. In the first part, the effect of the pertinent dimensionless parameters which govern the study such as Biot number (Bi), solid-fluid thermal conductivity ratio ( $\mathrm{Rc}$ ) and radius ratio $(\mathrm{Rr})$ on the LTNE intensity are analyzed by calculating: the local difference of temperature (LDT), the maximum of the local difference of temperature (LDT $\mathrm{max}_{\text {ax }}$ ) and the average of LDT. The results show that the increase of Biot number and the solid-fluid thermal conductivity ratio, and the decrease of radius ratio reduce the LTNE intensity. The intensity of the LDT in the developing region is greater than that of the fully developed region. In the second part, the convection heat transfer enhancement is studied, the results illustrate that the increase of $\mathrm{Bi}$, and $\mathrm{Rc}$, and the decrease of $\mathrm{Rr}$ represent good factors to ameliorate the rate of the convection heat transfer between the fluid and the inner wall.
\end{abstract}

Keywords: Porous medium; forced convection; local thermal non-equilibrium; heat transfer enhancement; Darcy- Brinkman- Forchheimer model.

\section{INTRODUCTION}

The thermal analysis of fluid flow through porous media is frequently encountered in many scientific fields and was studied by many researchers at various and different engineering applications such as electronic cooling, drying processes, thermal insulation, solar collectors, heat exchangers, nuclear reactors, chemical and petroleum engineering [1,2]. A review focused in the validity of the local thermal equilibrium fluid-solid and for the improvement of the convection heat transfer using porous material. Two primary models can be utilized for representing the convection heat transfer in a porous medium: local thermal equilibrium 
(LTE) model (i.e. one equation model) and (LTNE) model (i.e. two equations model). The LTNE incorporate the temperature difference between the fluid and solid phases. The assumption of LTE is not valid for some applications where a substantial temperature difference exists between the two phases [3].

Alazmi and Vafai [4] explored the characteristics of momentum and energy transport for free surface flows through porous media. It was showed that the LTNE between the two phases is found to be more pronounced when thermal dispersion effect is included. Khashan et al. [5] checked the validity of the local thermal equilibrium for different values of dimensionless parameters; they found that the low Peclet (Pe), Reynolds (Re), Darcy (Da) numbers and the increase of $\mathrm{Bi}$, Forchheimer coefficient were all found to improve the LTE validity condition. Moreover, the increase of the effective fluid-to-solid thermal conductivity ratio was found to extend the LTNE region. Marcelo B et al. [6] analyzed numerically the fully developed forced convection in a porous channel bounded by parallel plates based on a two-energy equation model. They found that high Re, low porosities, low particle diameters and low thermal conductivity ratios promote thermal equilibrium between phases, eventually leading to higher values of $\mathrm{Nu}$ for both the fluid and the solid phases. Felipe $\mathrm{T}$ et al. [7] studied an impinging jet on a flat plate covered with a layer of a porous material. The results indicated that for low porosities, low permeabilities, thin porous layers and for high thermal conductivity ratios. It was shown that the use of the LTNE model indicates that it is advantageous to use a layer of highly conducting and highly porous material attached to the hot wall. Also, Gazy F et al. [8] investigated the validity of the local thermal equilibrium assumption in the steady forced convection over a circular cylinder heated at constant temperature and embedded in a horizontal porous channel. Their results illustrated that the circumstances of a higher Re, a higher Prandtl number, a lower Da, a lower microscopic and macroscopic frictional flow resistance coefficient, a lower $\mathrm{Bi}$, a lower $\mathrm{Rc}$, a lower cylinderto-particle diameter ratio and a lower porosity, are identified as unfavorable circumstances for LTE condition to hold. Chanpreet et al. [9] investigated experimentally the validation of heat transfer models (LTE and LTNE) for unsteady flow of water through a tube filled with porous medium. Results show that the predictions of the two-equation model are uniformly superior to the one-equation model over the range of Peclet numbers between 500 and 4000 .

In the recent years a number of research papers interested on the improvement of the forced convection heat transfer by using porous media under LTE and LTNE assumptions [10-19]. For example, Bernardo et al. [10] solved analytically the forced convection in micro-channels filled with porous media under LTNE conditions. They found that the heat transfer increases as $\mathrm{Bi}$ increases and reaches asymptotic values. Yasser and Nader [11] numerically examined the heat transfer enhancement in a pipe partially filled with a porous medium. It is found that the optimum porous thickness for heat transfer enhancement with reasonable pressure drop is determined. Maziar Dehghan et al. [12] studied a thermally developing forced convective heat transfer inside a channel filled with a porous medium whose walls are imposed to a constant heat flux. The temperature difference between the fluid and solid phases (called the LTNE intensity) is analytically obtained. In addition, the Nusselt number of a thermally developing convection through a porous material is obtained for the LTNE condition. Heat transfer enhancement in a pipe or a channel partially or completely filled with porous medium was numerically investigated by Abdulmajeed [13]. It was shown that the partially filling of the conduit with porous medium has two advantages: it enhances the rate of heat transfer, and the pressure drop is much less than that for a conduit 
fully filled with a porous medium. Bogdan et al. [14] investigated experimentally and numerically the effect of metallic porous matrix inserted in a pipe on the rate of heat transfer. The pipe is subjected to a constant and uniform heat flux. It is shown that the heat transfer enhancement can be achieved using porous inserts whose diameters approach the diameter of the pipe and low permeability. Further improvement can be attained by increasing the thermal conductivity of the porous material. Targui [15] studied the fluid flow and heat transfer in a double pipe heat exchanger filled with porous media using the one energy equation model. This work discussed the optimal spacing, dimensions and the position of fixation of porous structures that enhance heat transfer performance. Fumei Rong et al. [16] investigated numerically the convection heat transfer inside pipe partially filled with porous medium. It was found that controlling the porous matrix thickness can significantly improve heat transfer performance and the influence of porosity is very small both on flow field and temperature field. Effects of variation of the thermal conductivity on forced convection in a parallel-plates channel heat exchanger occupied by a fluid saturated porous medium was investigated analytically by Maziar Dehghan [17]. It is found that a linear increase in the thermal conductivity of the medium results in a semi-linear increase in the Nusselt number. The heat transfer enhancement in an annular heat exchanger partially filled with porous media under LTE condition studied numerically by Chikh et al. [18]. It is shown that the use of porous substrate reduces the thermal entry length and leads to an increase in the heat transfer rate provided its thermophysical properties and thickness are well chosen. Akbarzadeh et al.[19] investigated numerically the combined effects of using nanofluid and wavy walls on the convection heat transfer inside heat exchanger filled with porous material. They found that the increase in the wave amplitude in the range of $0-0.1$ decreases the heat convection, while the increase of the wave amplitude in the range of 0.15-0.2 improves the convection heat transfer. The results, also, show that the Nusselt number can be improved by increasing the thickness of the porous layer and by decreasing the Darcy number.

The current study reveals that the examination of the intensity of the local thermal non-equilibrium for the phenomenon of forced convection heat transfer inside an annular pipe fully filled with fibrous metallic porous material is not well covered. Also, some pertinent parameters which govern the heat transfer improvement are not considered. Therefore, the present study aims to illustrate the effect of dimensionless parameters such as Biot number, thermal conductivity ratio and radius ratio on the intensity of the LTNE (i.e. validity of LTE). The improvement of the average Nusselt number under the LTNE assumption is discussed for different governing parameters to achieve high performance. 


\section{MATHEMATICAL FORMULATION}

\section{Problem Statement}

The geometry of the current study is shown in Figure1, in which the fluid with a uniform inlet velocity $U_{i n}$ and temperature $T_{i n}$ flows through an annular pipe completely filled with solid porous material of uniform porosity and permeability.The temperature of the inner wall Twis kept constant and the outer wall is thermally insulated. The main assumptions for this study are summarized as follows:

- $\quad$ The fluid flow is considered two-dimensional, steady state, laminar $(\operatorname{Re}<2300)$ and incompressible.

- $\quad$ The properties of the porous matrix are solid, isotropic and homogeneous.

- $\quad$ The fluid is Newtonian and its thermophysical properties are constant.

- Gravitational, viscous dissipation and thermal radiation effects are ignored.

- $\quad$ The study based on the local thermal non-equilibrium (LTNE) assumption.

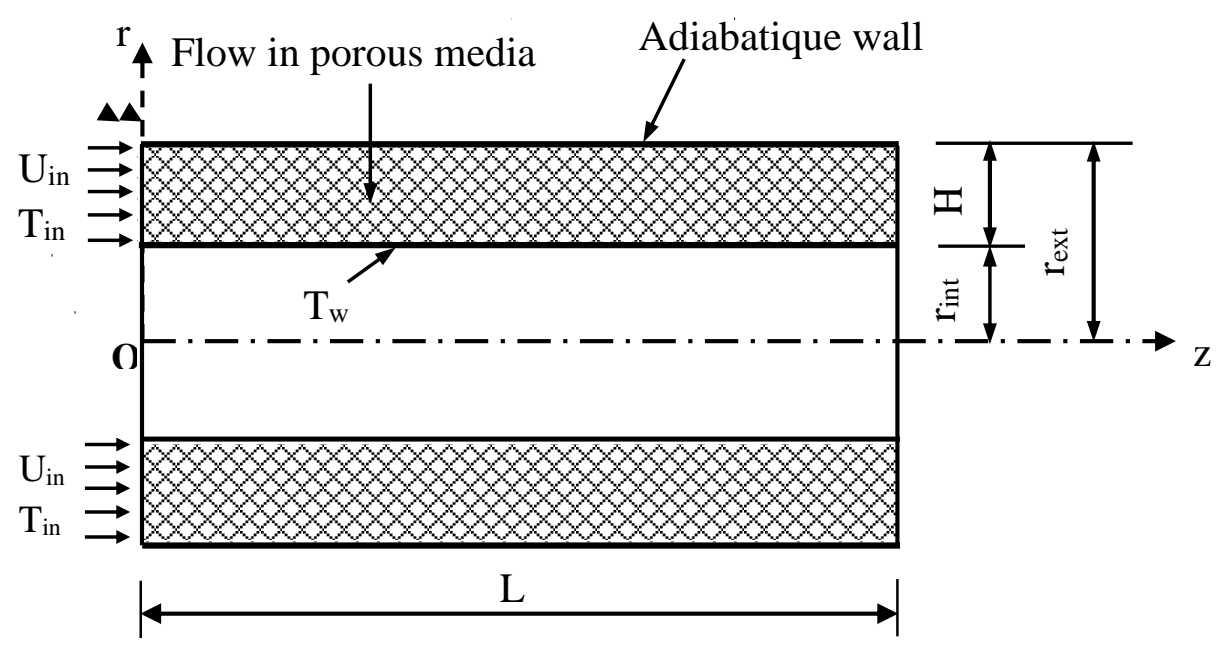

Figure 1. Schematic of the physical problem.

\section{Governing Equations}

The fluid flow in the porous matrix is modeled by using the Darcy-Brinkman-Forchheimer model. Under the above assumptions, the continuity and momentum equations presented in $[5,6,20]$ can be written in the following form:

- Continuity equation

$$
\frac{1}{r} \frac{\partial(\rho r v)}{\partial r}+\frac{\partial(\rho u)}{\partial z}=0
$$

- $\quad$ Momentum equation in axial direction (oz)

$$
\begin{aligned}
\frac{\rho}{\phi}\left[\frac{1}{r} \frac{\partial(\rho r v u)}{\partial r}+\frac{\partial(\rho u u)}{\partial z}\right] & =\mu\left[\frac{\partial}{\partial z}\left(\frac{\partial u}{\partial z}\right)+\frac{1}{r} \frac{\partial}{\partial r}\left(r \frac{\partial u}{\partial r}\right)\right]-\phi \frac{\partial p}{\partial z}-\phi \frac{\mu}{K} u \\
& -\frac{\rho \phi C_{F}}{K^{1 / 2}} \sqrt{u^{2}+v^{2}} u
\end{aligned}
$$


- $\quad$ Momentum equation in radial direction (or)

$$
\begin{aligned}
\frac{\rho}{\phi}\left[\frac{1}{r} \frac{\partial(\rho r v v)}{\partial r}+\frac{\partial(\rho u v)}{\partial z}\right]= & \mu\left[\frac{\partial}{\partial z}\left(\frac{\partial v}{\partial z}\right)+\frac{1}{r} \frac{\partial}{\partial r}\left(r \frac{\partial v}{\partial r}\right)-\frac{v}{r^{2}}\right]-\phi \frac{\partial p}{\partial r}-\frac{\mu \phi}{K} v \\
& -\frac{\rho \phi C_{F}}{K^{1 / 2}} \sqrt{u^{2}+v^{2}} v
\end{aligned}
$$

Where, $u$ and $v$ are the velocity components in axial and radial directions, respectively, $P$ is the intrinsic pressure of the fluid, $K$ and $\phi$ are the permeability and porosity of the porous medium, respectively, $\mu$ is dynamic viscosity, $\rho$ is density of the fluid, $C_{F}$ is the inertia coefficient of the porous matrix

\section{- $\quad$ Energy equation:}

Two differents models can be utilized for describing the convection heat transfer in porous media: the one-equation model and the two equation model. The one equation model (LTE model) is valid when the local temperature difference is negligible between the fluid and the solid phases, i.e. $\left(T_{f} \approx T s\right)$. The two-equation model (LTNE model) is used in some application when the local temperature difference between phases cannot be neglected, i.e. $\left(T_{f} \neq T s\right)[21]$. The present study is based on the two equation model (i.e. energy equation for the fluid phase and equation for the solid phase ). The LTNE model presented in references [11,20,22] can be written for this case as follow:

- $\quad$ Energy equation for fluid phase

$$
\frac{1}{r} \frac{\partial\left(\rho c_{p} r v T\right)}{\partial r}+\frac{\partial\left(\rho c_{p} u T\right)}{\partial z}=\frac{\partial}{\partial z}\left(\phi \lambda_{f} \frac{\partial T}{\partial z}\right)+\frac{1}{r} \frac{\partial}{\partial r}\left(r \phi \lambda_{f} \frac{\partial T}{\partial r}\right)+h_{s f} a_{s f}\left(T_{s}-T_{f}\right)
$$

- $\quad$ Energy equation for solid phase

$$
\left[\frac{1}{r} \frac{\partial}{\partial r}\left((1-\phi) \lambda_{s} r \frac{\partial T_{s}}{\partial r}\right)+\frac{\partial}{\partial z}\left((1-\phi) \lambda_{s} \frac{\partial T_{s}}{\partial z}\right)\right]-h_{s f} a_{s f}\left(T_{s}-T_{f}\right)=0
$$

Here, $T_{f}$ and $T_{S}$ are the temperature of fluid and solid phases, respectively, $h_{s f}$ is the fluid-solid heat transfer coefficient, $a_{s f}$ is the specific surface area (surface per unit volume), $C_{P}$ is the specific heat of the fluid, $\lambda_{f}$ and $\lambda_{S}$ are the thermal conductivity of the fluid and solid phases, respectively. Subscripts $f$ and $s$ are used for fluid and solid phases.

\section{Hydrodynamic and Thermal Boundary Conditions}

- $\quad$ Inlet (z=0): $u=u_{\text {in }}, \quad v=0, \quad T_{f}=T_{\text {in }}, T_{s}=T_{\text {in }}$

- Internal wall $\left(\mathrm{r}=\mathrm{r}_{\text {int }}\right): u=0, \quad v=0, \quad T_{f}=T_{w}, \quad T_{S}=T_{w}$

- $\quad$ External wall $\left(\mathrm{r}=\mathrm{r}_{\mathrm{ext}}\right): u=0, \quad v=0, \quad \frac{\partial T_{f}}{\partial r}=0, \frac{\partial T_{s}}{\partial r}=0$

- $\quad \operatorname{Outlet}(\mathrm{z}=\mathrm{L}): \frac{\partial u}{\partial z}=0, \frac{\partial v}{\partial z}=0, \frac{\partial T_{f}}{\partial z}=0, \frac{\partial T_{s}}{\partial z}=0$ 


\section{Generalization of the Mathematical Model}

In order to extract the dimensionless numbers and parameters that govern this study, it is necessary transformingthe mathematical model to the dimensionless form. This form can be obtained by introducing the following dimensionless variablesin the governing equations and boundary conditions:

$$
R=\frac{r}{D_{h}}, \quad Z=\frac{z}{D_{h}}, \quad U=\frac{u}{u_{i n}}, \quad V=\frac{v}{u_{i n}}, \quad \mathrm{P}=\frac{p}{\rho u_{i n}^{2}}, \quad \theta_{f}=\frac{T_{f}-T_{w}}{T_{i n}-T_{w}}, \theta_{S}=\frac{T_{S}-T_{w}}{T_{i n}-T_{w}}
$$

Where, $\mathrm{D}_{\mathrm{h}}$ is the hydraulic diameter, $D_{h}=2\left(r_{\text {ext }}-r_{\text {int }}\right)$

The system of equations from (1) to (5) has the following dimensionless form:

$$
\begin{gathered}
\frac{\partial U}{\partial Z}+\frac{1}{R} \frac{\partial}{\partial R}(R V)=0 \\
\frac{\partial}{\partial Z}(U U)+\frac{1}{R} \frac{\partial}{\partial R}(R V U)=\frac{\phi}{\operatorname{Re}}\left[\frac{\partial}{\partial Z}\left(\frac{\partial U}{\partial Z}\right)+\frac{1}{R} \frac{\partial}{\partial R}\left(R \frac{\partial U}{\partial R}\right)\right]-\phi^{2} \frac{\partial P}{\partial Z}-\frac{\phi^{2} U}{\operatorname{Re} D a} \\
-\frac{\phi^{2} C_{F}}{\sqrt{D a}} \sqrt{U^{2}+V^{2}} U \\
\frac{\partial}{\partial Z}(U V)+\frac{1}{R} \frac{\partial}{\partial R}(R V V)=\frac{\phi}{\operatorname{Re}}\left[\frac{\partial}{\partial Z}\left(\frac{\partial V}{\partial Z}\right)+\frac{1}{R} \frac{\partial}{\partial R}\left(R \frac{\partial V}{\partial R}\right)-\frac{V}{R^{2}}\right]-\phi^{2} \frac{\partial P}{\partial R} \\
\frac{\phi^{2} V}{\operatorname{Re} D a}-\frac{\phi^{2} C_{F}}{\sqrt{D a}} \sqrt{U^{2}+V^{2} V} \\
\left.\frac{\partial}{\partial Z}\left(U \theta_{f}\right)+\frac{1}{R} \frac{\partial}{\partial R}\left(R V \theta_{f}\right)=\frac{\partial}{\operatorname{Re} \operatorname{Pr}}\left[\frac{\partial \theta_{f}}{\partial Z}\right)+\frac{1}{R} \frac{\partial}{\partial R}\left(R \frac{\partial \theta_{f}}{\partial R}\right)\right]+\frac{B i \cdot R c}{\operatorname{Re} \cdot \operatorname{Pr}}\left(\theta_{S}-\theta_{f}\right) \\
{\left[\frac{\partial}{\partial Z}\left(\frac{\partial \theta_{S}}{\partial Z}\right)+\frac{1}{R} \frac{\partial}{\partial R}\left(R \frac{\partial \theta_{S}}{\partial R}\right)\right]-\left(\frac{B i}{1-\phi}\right)\left(\theta_{S}-\theta_{f}\right)=0}
\end{gathered}
$$

Here, Re, $\operatorname{Pr}, D a$ and Bi are Reynolds, Prandtl, Darcy and Biot numbers, respectively. Rc is the thermal conductivity ratio

$$
\operatorname{Re}=\frac{\rho u_{i n} D_{h}}{\mu}, \quad \operatorname{Pr}=\frac{\mu C_{P}}{\lambda_{f}}, \quad D a=\frac{K}{D_{h}^{2}}, \quad B i=\frac{h_{s f} a_{s f} D_{h}^{2}}{\lambda_{s}} \quad \text { and } \quad R c=\frac{\lambda_{S}}{\lambda_{f}}
$$


The boundary conditions in the dimensionless form become as follow:

- $\quad$ At the inlet $(\mathrm{Z}=0): U=1, \quad V=0, \quad \theta_{f}=1, \quad \theta_{S}=1$

- At the internal wall ( $\left.\mathrm{R}=\mathrm{R}_{\text {int }}\right): U=0, \quad V=0, \quad \theta_{f}=0, \quad \theta_{S}=0$

- At the external wall $\left(\mathrm{R}=\mathrm{R}_{\mathrm{ext}}\right): U=0, \mathrm{~V}=0, \frac{\partial \theta_{f}}{\partial R}=0, \frac{\partial \theta_{S}}{\partial R}=0$

- $\quad$ At the outlet $\left(Z=L^{*}=\mathrm{L} / \mathrm{D}_{\mathrm{h}}\right): \frac{\partial U}{\partial Z}=0, \frac{\partial V}{\partial Z}=0, \frac{\partial \theta_{f}}{\partial Z}=0, \frac{\partial \theta_{S}}{\partial Z}=0$

\section{Intensity of LDT (Intensity of LTNE)}

The LDT represent an important indicator to determine the appropriate model (LTE or LTNE model) for solving the problems of convection heat transfer in porous media. The LTE model is valid for very small values of LDT, while the LTNE model becomes the appropriate model and gives more accurate results for important values of LDT. The intensity of LDT (Intensity of LTNE) is analyzed by calculating LDT, LDT $_{\max }$ and ALDT, where:

- $\quad$ LDT: represent the local difference of dimensionless temperature between the solid and fluid phases at each node and is defined as:

$$
L D T(R, Z)=\left|\theta_{f}(R, Z)-\theta_{S}(R, Z)\right|
$$

- $\quad$ LDT $_{\max }$ represent the maximum local difference of dimensionless temperature over all the domain of flow and is defined as:

$$
L D T_{\max }=\max [L D T(R, Z)]
$$

- $\quad$ ALDT or LTNE: represent the Average of Local Difference of Temperature between fluid and solid phases over the computational domain, where LTNE is defined by Wong and Saeid [23]. as follow:

$$
\text { LTNE }=\frac{\sum_{1}^{N}\left|\theta_{f}-\theta_{S}\right|}{N}
$$

Where $N$ is the total number of nodes in the computational domain,

\section{Local and Average Nusselt Number}

The convection heat transfer coefficient between the inner wall and the moving fluid under the LTNE condition is determined as follow:

$$
h(z)\left[T_{w}-T_{f m}(z)\right]=-\left(\left.\phi \lambda_{f} \frac{\partial T_{f}}{\partial r}\right|_{r=r_{\mathrm{int}}}+\left.(1-\phi) \lambda_{S} \frac{\partial T_{s}}{\partial r}\right|_{r=r_{\mathrm{int}}}\right)
$$


Where, $h(z)$ is the local convection heat transfer coefficient, $T_{f m}(z)$ is the average temperature of the fluid at the transversal section $z$, and is defined as follows:

$$
T_{f m}(z)=\frac{\int_{r_{\text {int }}}^{r_{\text {ext }}} u T_{f} r d r}{\int_{r_{\text {int }}}^{r_{\text {ext }}} u r d r}
$$

Writing equation (14) in dimensionless form, we get the local Nusselt number as follow:

$$
N u(Z)=\frac{h(z) D_{h}}{\lambda_{f}}=\frac{-\left.\phi \frac{\partial \theta_{f}}{\partial R}\right|_{R=R_{\mathrm{int}}}-\left.(1-\phi) R c \frac{\partial \theta_{s}}{\partial R}\right|_{R=R_{\mathrm{int}}}}{\theta_{f m}(Z)-1}
$$

Where, $\theta_{f m}(Z)$ is the dimensionless bulk temperature of the fluid and is defined:

$$
\theta_{f m}(Z)=\frac{\int_{R_{\text {int }}}^{R_{\text {ext }}} U \theta_{f} R d R}{\int_{R_{\text {int }}}^{R_{\text {ext }}} U R d R}
$$

The rate of the convection heat transfer is evaluated by calculating the average Nusselt number $\overline{N u}$, which is expressed as:

$$
\overline{N u}=\frac{\bar{h} D_{h}}{\lambda_{f}}=\frac{1}{L^{*}} \int_{0}^{L^{*}} N u(Z) d Z
$$

\section{NUMERICAL SOLUTION}

\section{Procedure of Solution}

The dimensionless governing equations (continuity, momentum and energy) are transformed to a set of algebraic equations by using the finite volume method. The Power Law Differencing Scheme (PLDS) is applied to treat the diffusion and convection terms of governing equations $[15,24]$. The algebraic equations resulted from the discretization of the differential equations is written as follow:

$$
A_{i j} \Phi_{i j}=A_{i-1, j} \Phi_{i-1, j}+A_{i+1, j} \Phi_{i+1, j}+A_{i, j-1} \Phi_{i, j-1}+A_{i, j+1} \Phi_{i, j+1}+b
$$

Where: $\Phi=U, V, \theta_{f}, \theta_{S}, P^{*}$ (Pressure correction )

The numerical method used in the present study is based on the algorithm SIMPLE (Semi-Implicit Method for Pressure-Linked Equations) of Patankar and Spalding [24].The system of the algebraic equations is solved iteratively by using the line by line procedure in conjunction with the tri-diagonal matrix algorithm (TDMA). This iterative solution needs under relaxation to control the changes in the values of all the variables during iteration $[25,26]$. 
The solution is considered obtained and the computation can be stopped at the iteration at which the following conditions are achieved:

$$
\left\{\begin{array}{l}
\max \left|R_{\Phi}\right|<10^{-6} \\
\sum\left|R_{\Phi}\right|<10^{-4}
\end{array} \quad \text { where: } \quad \Phi=U, V, \theta_{f}, \theta_{S}, P^{*}\right. \text { (Pressure correction) }
$$

Where:

$$
R_{\Phi i j}=A_{i j} \Phi_{i j}-A_{i-1, j} \Phi_{i-1, j}-A_{i+1, j} \Phi_{i+1, j}-A_{i, j-1} \Phi_{i, j-1}-A_{i, j+1} \Phi_{i, j+1}-b_{i j}
$$

$R_{\Phi i j}$ : represent the residual of the dicretized algebraic equation at each control volume and for each variable.

The convergence of the numerical solution is monitored by tracking the values of the average Nusselt number with iterations as presented in Figure2.

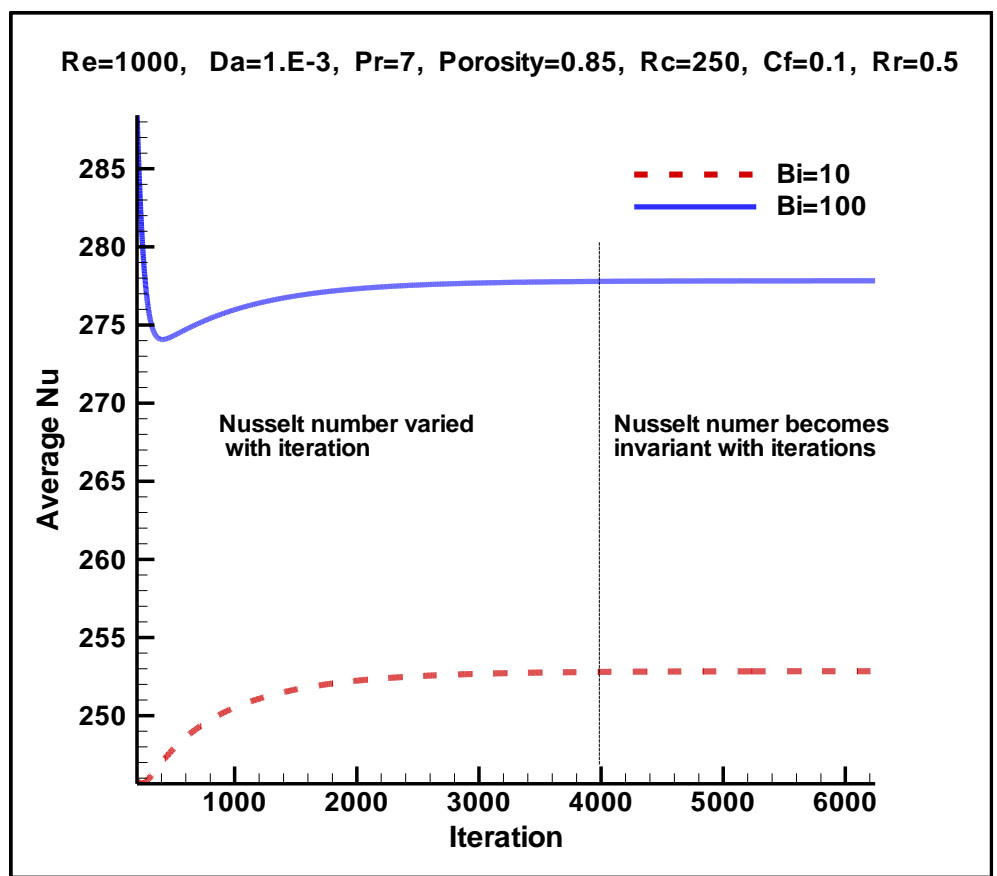

Figure 2. Convergence of the numerical solution

\section{Numerical Validation}

The validation of the method of solution used in this study is tested by comparing the Nusselt number of the present method with other studies based on the Darcy-Brinkman-Forchheimer model. The first comparison was done with the solution of Abdulmajeed [13], and illustrated in Table 1 for the case of the laminar forced convection in pipe fully filled with porous media at different permeability (different Darcy number). The comparison shows that the maximum difference as relative error is not exceed $0.86 \%$. The second comparison was done with the solution of Chikh [18] and presented in the Figure 3 for the case of an annular pipe filled with porous media at different thickness of porous material. Excellent agreement is found between the present numerical results and results given in [18]. Hence, from the two comparisons the present method which based on the SIMPLE algorithm is valid to simulate flow and heat transfer in porous media at wide range of hydrodynamic and thermal parameters for new geometry and conditions. 
Table 1.Comparison of the Nusselt number obtained by the numerical solution with the solution presented in [13] at different values of Darcy number

\begin{tabular}{llllllll}
\hline $\mathrm{Da}$ & $10^{-3}$ & $2.10^{-3}$ & $3.10^{-3}$ & $5.10^{-3}$ & $10^{-2}$ & $2.10^{-2}$ & $5.10^{-2}$ \\
\hline Nu present work & 5.436 & 5.299 & 5.199 & 5.049 & 4.8 & 4.53 & 4.195 \\
Relative error (\%) with[13] & 0.15 & 0.19 & 0.21 & 0.26 & 0.19 & 0.33 & 0.72 \\
\hline
\end{tabular}

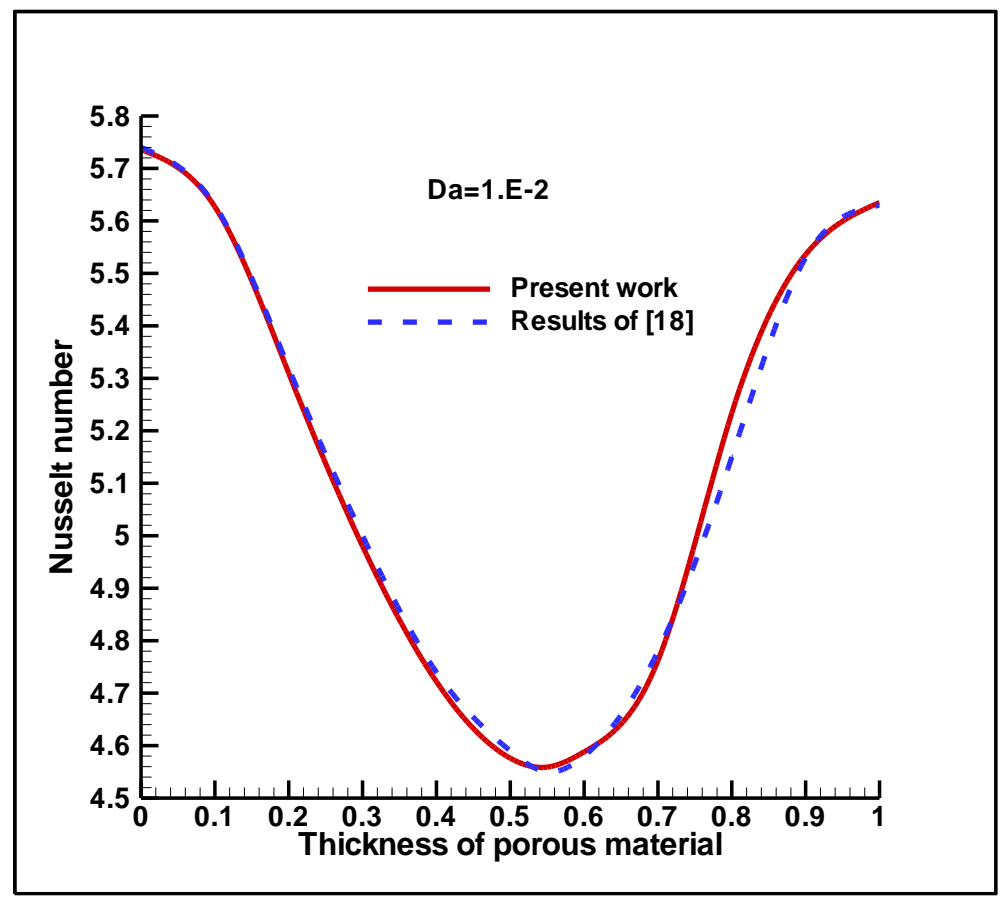

Figure3. Comparison of the present fully developed Nusselt number versus the thickness of the porous media with the solution of Chikh [18]

\section{Choice of Grid}

The choice of the grid of the computational domain is based on the study of the independence between the grid and the numerical solutions. In this case the independence (grid - solution) was performed for six meshes $(\mathrm{Z}, \mathrm{R}) 61 * 31,81 * 41,101 * 51,121 * 61,141 * 71$ and $161 * 81$. The meshes are tested by the calculation of the Nusselt number $(\mathrm{Nu})$ values of laminar flow in an annular pipe presented in Tables 2 and 3 in two different cases (empty and fully filled annular pipe with porous media). For the case of the empty pipe, the results presented in Table2 show that the Nusslet number is constant for the last three grids. In the case of the fully filled pipe, the Table 3 shows that the differences between the values of $\mathrm{Nu}$ are not noticeable and there is a great affinity between the last three meshes. The increase in the mesh more than $\left(121^{*} 61\right)$ increases the run time without any amelioration in the accuracy of solution. Hence, the grid $\left(121^{*} 61\right)$ is sufficient enough for the simulation of this problem. 
Table 2.Nusselt number of empty annular pipe (without porous media)

\begin{tabular}{lllllll}
\hline Mesh & $61 * 31$ & $81 * 41$ & $101 * 51$ & $121 * 61$ & $141 * 71$ & $161 * 81$ \\
\hline $\mathrm{Nu}$ & 5.7341 & 5.7358 & 5.7366 & 5.737 & 5.737 & 5.737 \\
\hline
\end{tabular}

Table 3.Nusselt number of fully filled annular pipe with porous media at: $\operatorname{Re}=500, \operatorname{Pr}=7, \operatorname{Rc}=250$ and $\mathrm{Da}=10^{-3}$

\begin{tabular}{lllllll}
\hline Mesh & $61 * 31$ & $81 * 41$ & $101 * 51$ & $121 * 61$ & $141 * 71$ & $161 * 81$ \\
\hline $\mathrm{Nu}$ & 214.12 & 214.25 & 214.32 & 214.35 & 214.37 & 214.39 \\
\hline
\end{tabular}

\section{RESULTS AND DISCUSSION}

In all the calculations of the present study the Forcheimer coefficient, porosity and the computational domain are kept constant. Where: $\mathrm{CF}=0.1$ (for the case of foam metal fibers) [20], $=0.85$ and $\mathrm{L}^{*}=\mathrm{L} / \mathrm{Dh}=10$.

\section{Effect of Biot Number on the LDT Intensity}

The Biot number is a direct reflection of the heat transfer coefficient in the pores between fluid and solid matrix. The effect of Bi on the intensity of The LTNE is studied by the presentation of LDT profiles in Figure 4 at two distinct axial positions $\mathrm{Z}=0.25 \mathrm{~L}^{*}$ and $\mathrm{Z}=0.75 \mathrm{~L}^{*}$ which represents the developing and fully developed regions, respectively. In this simulation $\mathrm{Bi}$ varied between 0.1 and 1000. The results show that the effect of $\mathrm{Bi}$ on the values of LDT is considerable, where the LDT decreases by increasing $\mathrm{Bi}$, this decrease indicates that the two phases are trend to the LTE especially for high Bi. The diminution of LDT between the fluid and solid matrix is due to the augmentation of interstitial heat transfer coefficient hsf which approaches the temperatures of the two phases. It is seen in the Figure 4 that the LDT profiles are influenced by the axial position this due to the fact that the convection heat transfer at $\mathrm{Z}=0.25 \mathrm{~L}^{*}$ is larger than that in the fully developed region at $\mathrm{Z}=0.75 \mathrm{~L}^{*}$. Also the LDT intensity is revealed by plotting the LDT contours at different values of $\mathrm{Bi}$ as presented in Figure 5. The contours illustrate that The LTE condition (LDT negligible) can be obtained in the fully developed region at medium values $\mathrm{Bi}(\mathrm{Bi}>10)$. While in the developing region the high values of $\mathrm{Bi}(\mathrm{Bi}>100)$ are necessary to attain the LTE. Also it can be observed that the LDT peak appears in a zone localized at the lower left corner of the pipe at which characterized by important convection heat transfer and is adjacent to the active wall. This zone becomes very small by increasing $\mathrm{Bi}$ and vanishes at higher $\mathrm{Bi}$ which makes all the domain of flow in LTE. When the LTE is dominant, the Nusselt number of the LTNE model becomes very close to that calculated from LTE model as presented in Table 4.

Table 4.Nusselt number as a function of Bi for LTNE and LTE models at: $\mathrm{Re}=500, \mathrm{Pr}=7, \mathrm{Rc}=250$ and $\mathrm{Da}=10-3$

\begin{tabular}{lllllllllll}
\hline $\mathrm{Bi}$ & 0.01 & 0.05 & 0.1 & 0.5 & 1 & 5 & 10 & 50 & 100 & 500 \\
\hline $\mathrm{Nu}$ (LTNE) & 29.3 & 35.9 & 43.7 & 89.9 & 125.1 & 207.3 & 226.7 & 243.7 & 245.7 & 246.9 \\
$\mathrm{Nu}$ (LTE) & & & & & 245,2 & & & & & \\
\hline
\end{tabular}




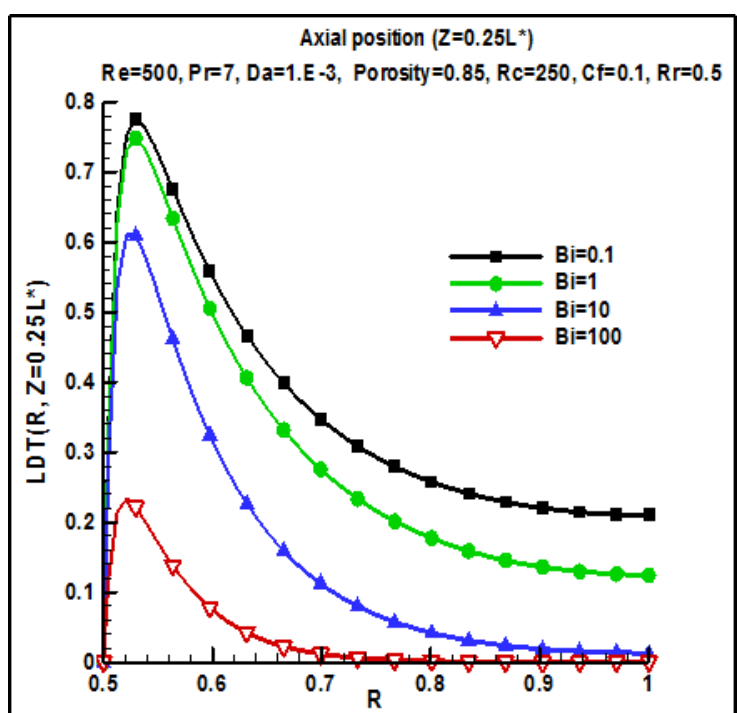

(a) $\mathrm{Z}=0.25 \mathrm{~L} *$

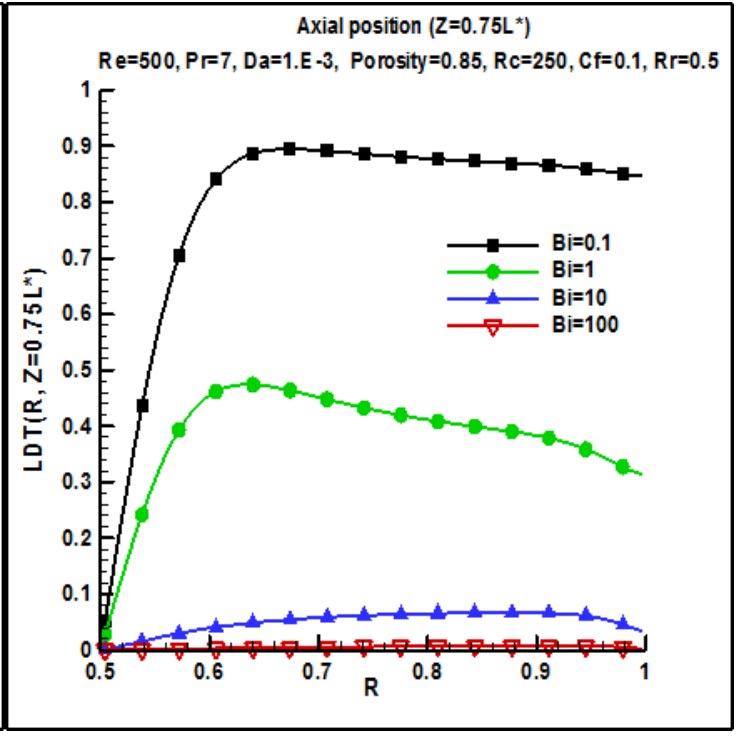

(b) $\mathrm{Z}=0.75 \mathrm{~L}^{*}$

Figure 4. Effect of Biot number on the LDT profiles at two different axial positions: (a) $\mathrm{Z}=0.25 \mathrm{~L}^{*}$; (b) $\mathrm{Z}=0.75 \mathrm{~L}^{*}$.

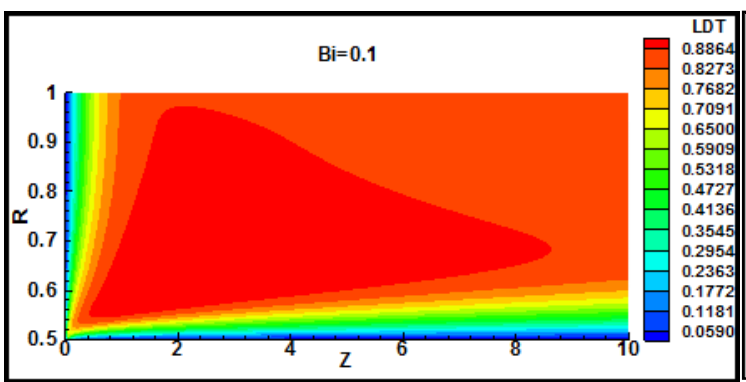

(a)

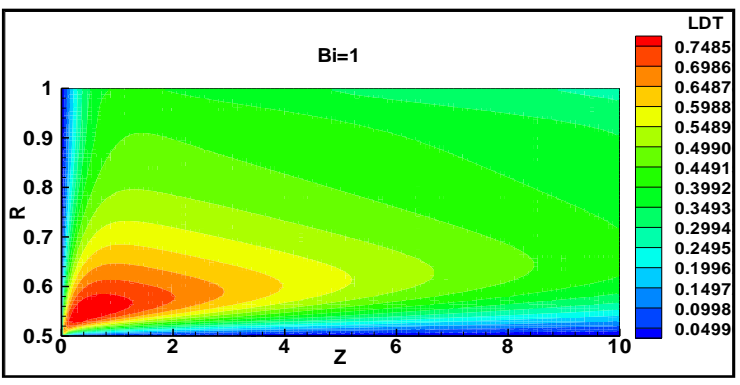

(b)

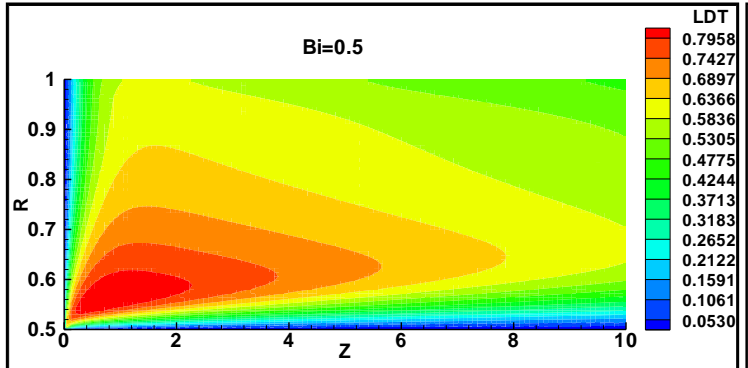

(c)

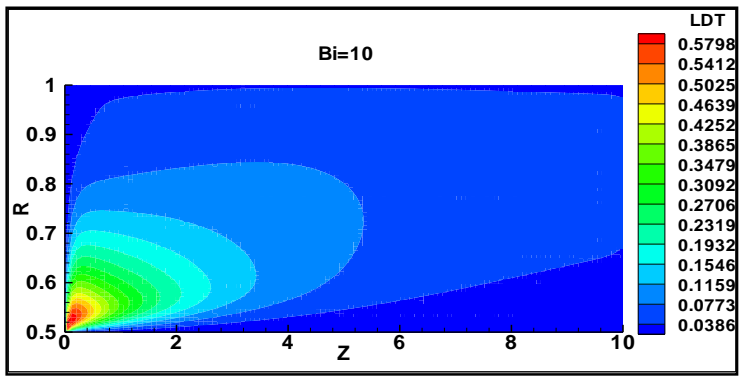

(d) 


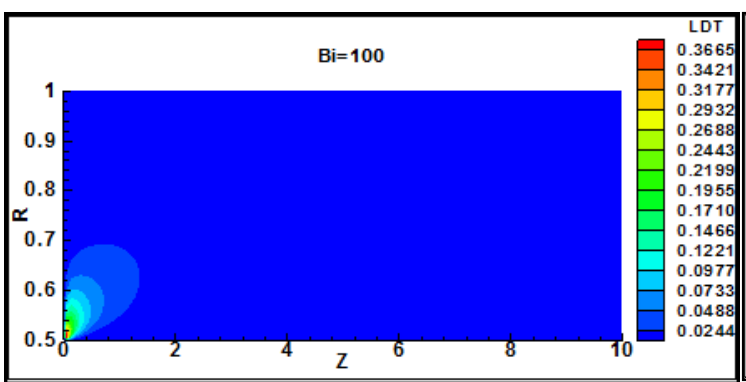

(e)

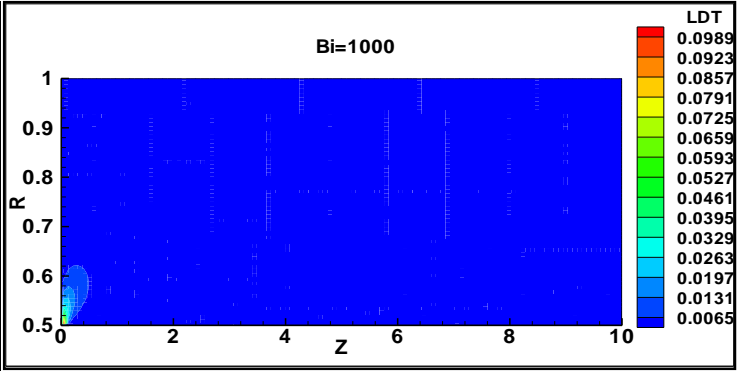

(f)

Figure 5. Contours of the LDT for different Biot numbers: (a) $\mathrm{Bi}=0.1$, (b) $\mathrm{Bi}=0.5$, (c) $\mathrm{Bi}=1$, (d) $\mathrm{Bi}=10$, (e) $\mathrm{Bi}=100$ and (f) $\mathrm{Bi}=1000$.

\section{Effect of the Thermal Conductivity Ratio and Radius Ratio on the LDT Intensity}

In order to reveal the effect of the radius ratio and the thermal conductivity ratio on the LDT intensity, the variations of the $\mathrm{LDT}_{\max }$ and ALDT versus radius ratio (Rr) are presented in Figures 6 and 7 for $R r$ varied from 0.1 to 0.9 and for different values of thermal conductivity ratio $(1,250$ and 500$)$. It can be seen that:

- $\quad$ The LDT intensity is inversely proportional to the solid-fluid thermal conductivity ratio Rc, where the $\mathrm{LDT}_{\max }$ and ALDT decrease by increasing Rc. This is due to the fact that the increase of $\mathrm{Rc}$ ameliorate the heat conduction in the porous matrix which reduces the local difference of temperature between the two phases.

- The LDT intensity is proportional to the radius ratio $\mathrm{Rr}$, where the $\mathrm{LDT}_{\max }$ and ALDT increase as $\mathrm{Rr}$ increases. The physical interpretation of this phenomenon is for fixed Reynolds number, the increase of $\mathrm{Rr}$ leads to decrease the hydraulic diameter (i.e. decrease in the area of annular cross section), which accompanied by the augmentation of dimensional velocity. As proved above the increase of the velocity makes the LTNE more dominate. Consequently, the increase of $\mathrm{Rr}$ is not suitable to achieve the local thermal equilibrium.

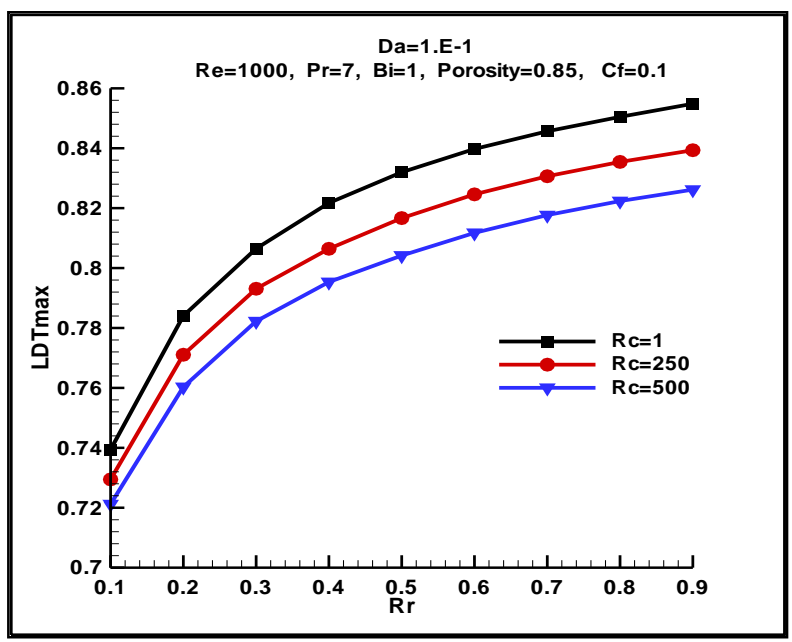

(a) $\mathrm{Da}=10-1$

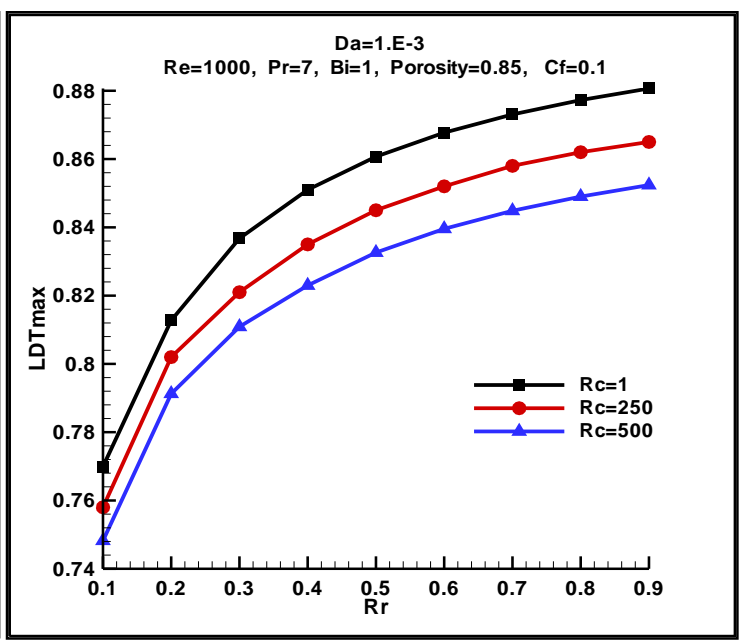

(b) $\mathrm{Da}=10-3$

Figure 6. The LDTmax as a function of radius ratio for different thermal conductivity ratio: (a) $\mathrm{Da}=10-1$, (b) $\mathrm{Da}=10-3$. 


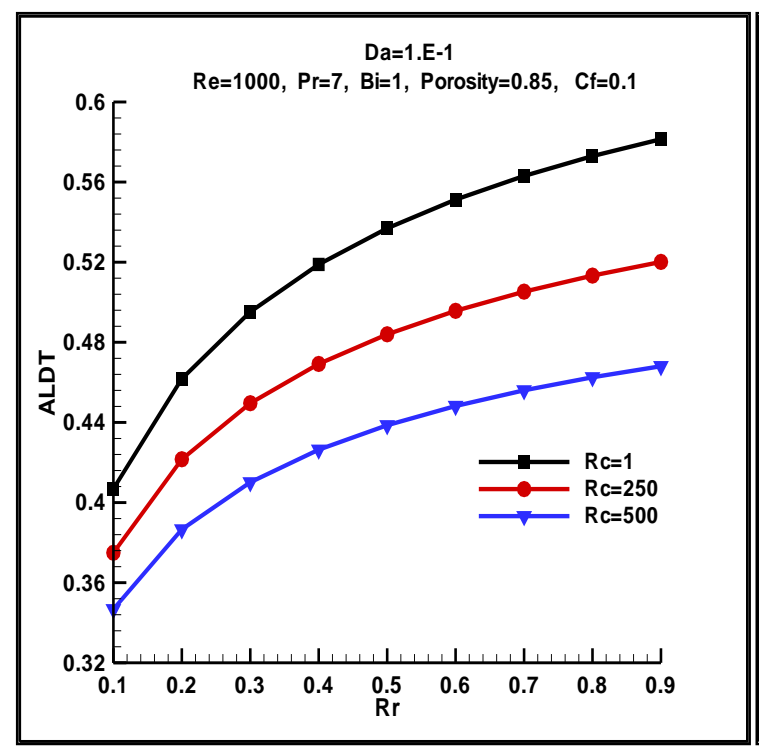

(a) $\mathrm{Da}=10-1$

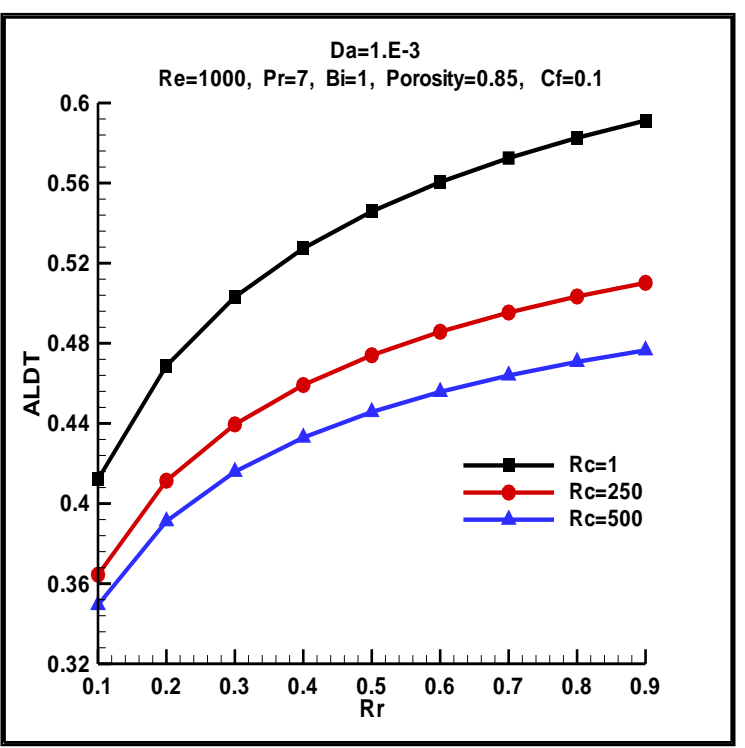

(b) $\mathrm{Da}=10-3$

Figure 7. ALDT as a function of radius ratio for different thermal conductivity ratio: (a)

$$
\mathrm{Da}=10-1 \text {, (b) } \mathrm{Da}=10-3
$$

\section{Effect of Biot Number on the Average Nusselt Number}

The effect of $\mathrm{Bi}$ on the rate of heat transfer is studied by calculating the average Nusselt number, for $\mathrm{Bi}$ varied from $10^{-1}$ to $10^{4}$ at different $\operatorname{Re}(500,1000,1500)$. The results are plotted in Figure 8 at two different values of Darcy. In the range of the operating parameters which used in the calculations, the influence of $\mathrm{Bi}$ number on the average $\mathrm{Nu}$ can be divided in two cases:

- $\quad$ Zone of LTNE ( $\mathrm{Bi}<100)$ : in this case there is no thermal equilibrium between the solid and fluid phases. It is clear that the Bi number has an important effect on the rate of convective heat transfer, where the average $\mathrm{Nu}$ increases by increasing $\mathrm{Bi}$ until the LDT between the two phases becomes negligible. The increase of Bi number (i.e. increase of the interstitial convection heat transfer coefficient $h_{\text {sf }}$ ) improves the rate heat transfer by convection between the internal wall of the pipe and the moving fluid.

- $\quad$ Zone of LTE $(\mathrm{Bi}>100)$ : in this case the average Nu reaches the maximum value and takes an asymptote form, this means that when the LTE is dominate over all the flow domain, the heat transfer between the internal wall of the pipe and the moving fluid is not influenced by the augmentation of $\mathrm{Bi}$ number. This due to the fact that when the local thermal equilibrium is occurred, the interstitial heat transfer (fluid-solid in the pores) becomes very weak. These results have the similar trends with the solution of [10] although the physical geometries are not the same.

From these results, it can be concluded that the variation of the average $\mathrm{Nu}$ as a function of $\mathrm{Bi}$ is one of the criteria for distinguishing between the LTNE and LTE zones such that the beginning of the stability of average $\mathrm{Nu}$ indicates that the thermal equilibrium between the two phases is reached. It can be observed also that the effect of Reynolds number on the average $\mathrm{Nu}$ increases by increasing $\mathrm{Bi}$. Therefore, the influence of Reynolds on the rate of heat transfer by convection in the LTE zone (i.e. high Bi) is more important in comparison with its effect on the LTNE zone ((i.e. low Bi) as shown in Figure 8. 


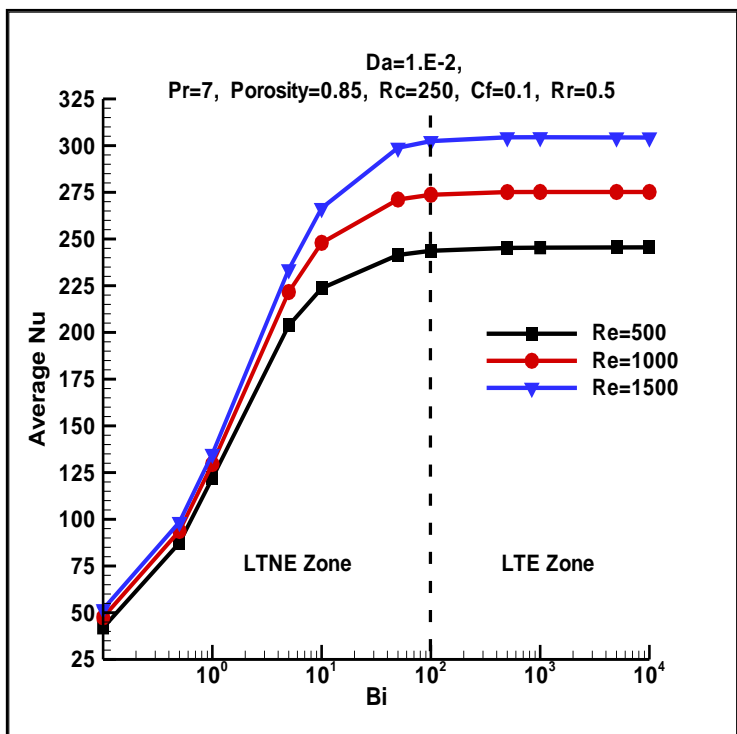

(a) $\mathrm{Da}=10-2$

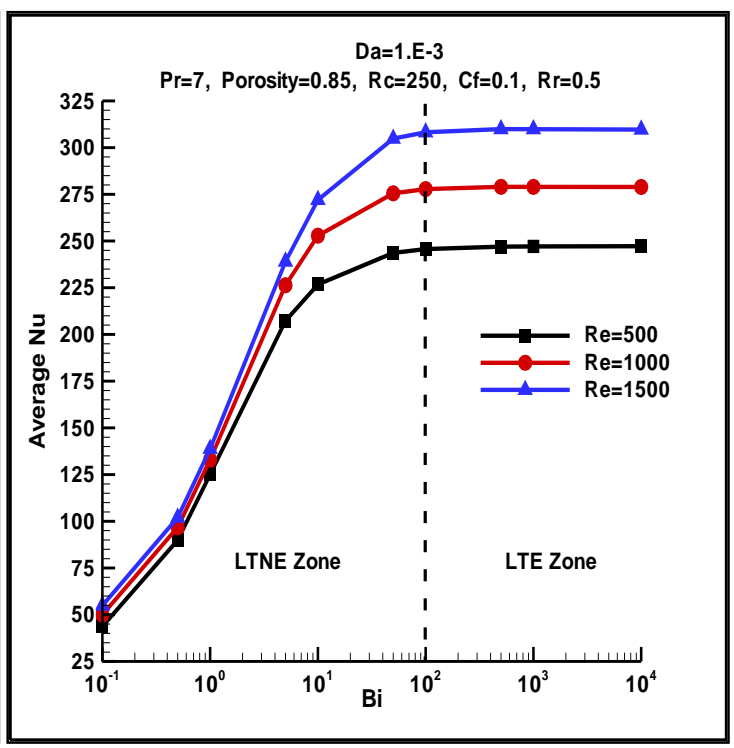

(b) $\mathrm{Da}=10-3$

Figure 8. Average Nusselt number as a function of Biot for different values of Re:

(a) $\mathrm{Da}=10-2$, (b) $\mathrm{Da}=10-3$.

\section{Effect of the Thermal Conductivity Ratio and Radius Ratio on the Average Nusselt Number}

Figure 9, illustrate the combined effect of the solid-fluid thermal conductivity ratio $\mathrm{R}_{\mathrm{C}}$ and the radius ratio $\mathrm{Rr}$ on the average $\mathrm{Nu}$, where $\mathrm{Rr}$ is varied from 0.1 to 0.9 at different $\mathrm{Rc}(150$, $200,250)$. The results are obtained at $\operatorname{Re}=1000, \operatorname{Pr}=7, \mathrm{Bi}=1$. It is found that:

- The increase of the $\mathrm{Rr}$ of the annular pipe decreases the average convection heat transfer, where the maximum values of the $\mathrm{Nu}$ are obtained at the lower $\mathrm{Rr}$. This is due to the fact that for fixed value of $\mathrm{Re}$, the increase of $\mathrm{Rr}$ reduces the dimensional velocity of the flow which leads to decrease average $\mathrm{Nu}$.

- The convection heat transfer between the inner wall and moving fluid is significantly affected by the values of Rc, where the greater value of average $\mathrm{Nu}$ is obtained at higher Rc, this implications is due to the increase in the thermal conductivity of porous matrix which ameliorate the conduction heat between the porous medium and the active wall of the annular pipe. Therefore for the case of heating (Tw>Tin), the fluid absorbs simultaneously the heat from the active wall and the porous matrix which leads to the augmentation of the average Nusselt number. 


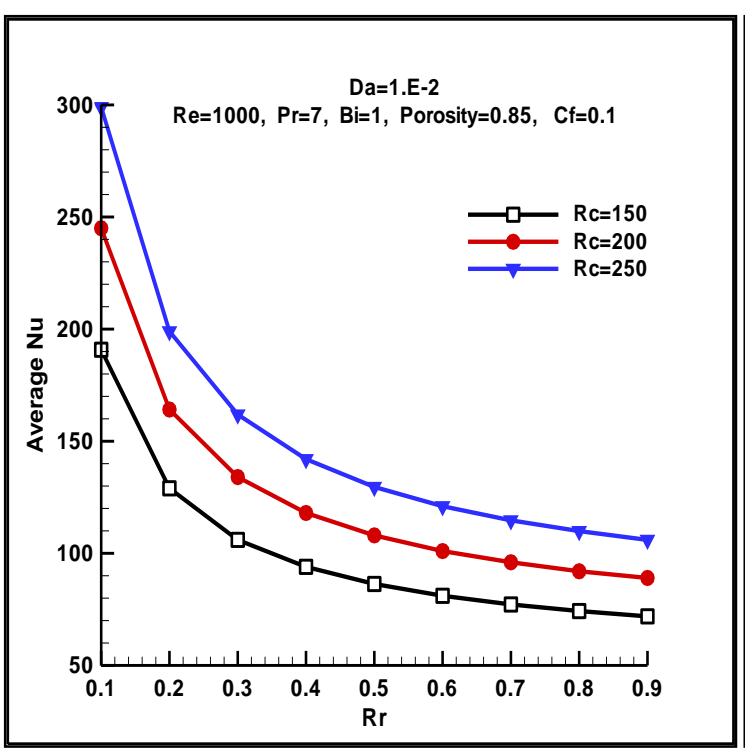

(a) $\mathrm{Da}=10-2$

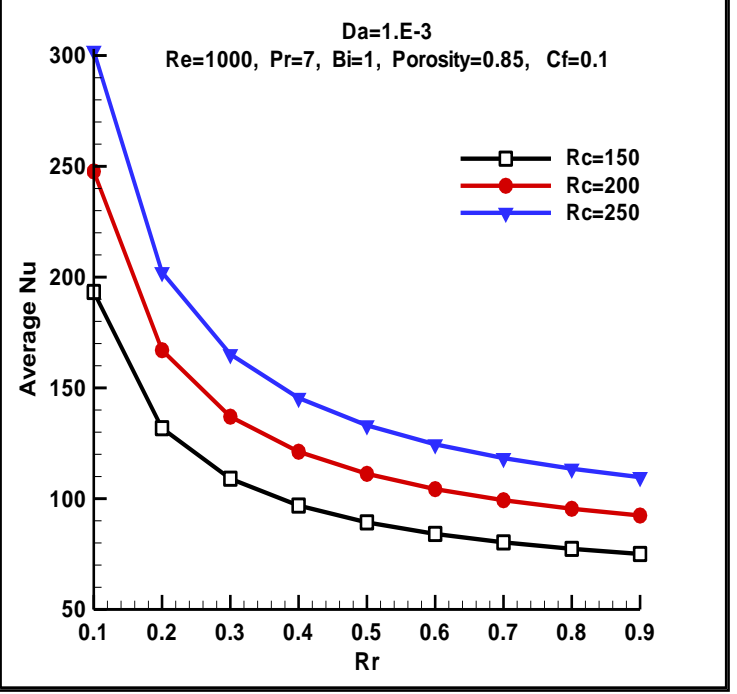

(b) $\mathrm{Da}=10-3$

Figure 9. Average Nusselt number as a function of Rr for different Rc:

(a) $\mathrm{Da}=10-2$, (b) $\mathrm{Da}=10-3$.

\section{CONCLUSIONS}

In this study, thermal analysis through an annular pipe completely filled with porous media based on a two-energy equation model is investigated numerically. Effects of pertinent dimensionless parameters on the validity of the LTE and the improvement of the heat transfer are analyzed and discussed. In the range of the operating parameters which used in this simulation and from the results obtained in this study, it can be concluded that:

- $\quad$ The increase of $\mathrm{Bi}$ and $\mathrm{Rc}$, and the decrease of the $\mathrm{Rr}$ represent principal parameters to reduce the LDT intensity and support the validity of the LTE condition. Consequently, the one model equation can be used to simulate the convection heat transfer in porous media for higher values of $\mathrm{Bi}$ and $\mathrm{Rc}$, and for lower values of radius ratio $\mathrm{Rr}$.

- $\quad$ The using of the LTE assumption is very limited and the LTNE assumption is more practically and accurate.

- $\quad$ The increase of Bi represents the important parameter to attain the LTE, where for high Bi number; the influence Rc and Rr on the LTNE intensity becomes very small.

- The LTE condition can be rapidly achieved in the fully developed region in comparison with the developing region (i.e. entrance of the pipe).

- $\quad$ The increase of $\mathrm{Bi}$ and $\mathrm{Rc}$, and the decrease of $\mathrm{Rr}$ represent good factors to ameliorate the convection heat transfer, where these conditions lead to increase the average Nusselt number

- For low values of $\mathrm{Bi}$, the effect of $\mathrm{Re}$ on the average $\mathrm{Nu}$ is not significant and becomes more significant by increasing $\mathrm{Bi}$. Therefore, the influence of $\mathrm{Re}$ on the rate of heat transfer by convection in the LTE case is more important than its effect on the LTNE case. 


\section{REFERENCES}

[1] Bader A, Kambiz V. Analysis of fluid flow and heat transfer interfacial conditions between a porous medium and a fluid layer. International Journal of Heat and Mass Transfer. 2001;44:1735-1749.

[2] Zallama B, Zili G, Nasrallah SB. Forced convection in a cylinder filled with porous medium, including viscous dissipation effects. Journal of Applied Fluid Mechanics. 2016;9:139-145.

[3] Bader A, Kambiz V. Analysis of variants within the porous media transport models.ASME Journal of Heat Transfer. 2000;122:303-326.

[4] Bader A, Kambiz V. Analysis of variable porosity, thermal dispersion, and local thermal non equilibrium on free surface flows through porous media. ASME Journal of Heat Transfer. 2004;126:389-399.

[5] Khashan SA, Al-Amiri AM, Al-Nimr MA. Assessment of the local thermal nonequilibrium condition in developing forced convection flows through fluid-saturated porous tubes. Applied Thermal Engineering. 2005;25:1429-1445.

[6] Marcelo BS, Marcelo JSDL. Laminar heat transfer in a porous channel simulated with a two-energy equation model. International Communication of Heat and Mass Transfer. 2009;36:1002-1007.

[7] Felipe TD, Marcelo JSDL. Simulation of laminar impinging jet on a porous medium with a thermal non-equilibrium model. International Journal of Heat and Mass Transfer. 2010;53:5089-5101.

[8] Gazy FAS, John S, Mark CT. Validation of thermal equilibrium assumption in forced convection steady and pulsatile flows over a cylinder embedded in a porous channel. International Communication of Heat and Mass Transfer. 2013;43:30-38.

[9] Chanpreet S, Tathgir RG, Muralidhar K. Experimental validation of heat transfer models for flow through a porous medium. Heat and Mass Transfer. 2006;43:55-72.

[10] Bernardo B, Oronzio M, Guy L. Forced convection in micro-channels filled with porous media in local thermal non-equilibrium conditions. International Journal of Thermal Science. 2014;77:206-222.

[11] Yasser M, Nader K. Numerical investigation of heat transfer enhancement in a pipe partially filled with a porous material under local thermal non-equilibrium condition. International Journal of Heat and Mass Transfer. 2014;68:161-173.

[12] Maziar D, Valipour MS, Amir K, Saedodin S, Nima S. On the thermally developing forced convection through a porous material under the local thermal non-equilibrium condition: An analytical study.International Journal of Heat and Mass Transfer. 2016;92:815-823.

[13] Abdulmajeed AM. Heat transfer enhancements in heat exchangers fitted with porous media Part I: constant wall temperature. International Journal of Thermal Science. 2003;42:385-395.

[14] Bogdan IP, Abdulmajeed AM. An experimental and numerical study on heat transfer enhancement for gas heat exchangers fitted with porous media. International Journal of Heat and Mass Transfer. 2004;47:4939-4952.

[15] Targui N, Kahalerras H. Analysis of fluid flow and heat transfer in a double pipe heat exchanger with porous structures. Energy Conversion and Management. 2008;49:3217-3229. 
[16] Fumei R, Wenhuan Z, Baochang S, Zhaoli G. Numerical study of heat transfer enhancement in a pipe filled with porous media by axisymmetric TLB model based on GPU. International Journal of Heat and Mass Transfer. 2014;70:1040-1049.

[17] Maziar D, Mohammad SV, Seyfolah S. Temperature-dependent conductivity in forced convection of heat exchangers filled with porous media: A perturbation solution. Energy Conversion and Management. 2015;91:259-266.

[18] Chikh S, Boumedien A, Bouhadef K, Guy L. Heat transfer enhancement by porous substrate addition on the inner wall of a tubular heat exchanger. Revue Générale de Thermique. 1997;36:41-50.

[19] Akbarzadeh M, Rashidi S, Karimi N, Omar N. First and second laws of thermodynamics analysis of nanofluid flow inside a heat exchanger duct with wavy walls and a porous insert. Journal of Thermal Analysis and Calorimetry. 2019;135:177-194.

[20] Nield DA, Bejan A .Convection in porous media. 4ed, Springer, New York, 2013.

[21] Dae-Young L, Kambiz V. Analytical characterization and conceptual assessment of solid and fluid temperature differentials in porous media. International Journal of Heat and Mass Transfer. 1999;42:423-435.

[22] Gazy FAS, Akira N, John S, Mark CT. The effect of porous media particle size on forced convection from a circular cylinder without assuming local thermal equilibrium between phases. International Journal of Heat and Mass Transfer. 2012; 55:3366-3378.

[23] Wong KC, Saeid NH. Numerical study of mixed convection on jet impingement cooling in a horizontal porous layer under local thermal non-equilibrium conditions. International Journal of Thermal Sciences. 2009;48:860-870.

[24] Patankar SV. Numerical heat transfer and fluid flow. McGraw-Hill, New York 1980.

[25] Saeid NH. Numerical predictions of sand erosion in a choke valve. Journal of Mechanical Engineering and Sciences. 2018;12:3988-4000.

[26] Sadouk HC, Chikh S, Guy L. Numerical Modeling of a Turbulent Flow in a Flat Channel with a Fibrous Coating on the Walls. Journal of Applied Fluid Mechanics. 2019;12:1-8. 